\title{
Variabilidade espacial das concentrações de nutrientes foliares da soja correlacionadas com atributos químicos de um Latossolo Vermelho distroférrico ${ }^{1}$
}

\author{
Rodolfo de Niro Gazola ${ }^{2}$, Lenon Henrique Lovera², Thiago de Souza Celestrino', Raíssa Pereira Dinalli ${ }^{2}$, \\ Rafael Montanari², Hernandes Andrade Queiroz ${ }^{2 *}$
}

10.1590/0034-737X201764040014

\section{RESUMO}

O estudo dos atributos do solo e da planta, por meio da geoestatística e da consequente geração de mapas de manejo, resulta na utilização racional de fertilizantes e, assim, na redução de custos para o produtor. No ano agrícola de 2013/2014, no município de Selvíria, MS, objetivou-se, com este estudo, avaliar as dependências linear e espacial dos atributos $\mathrm{Ca}, \mathrm{Mg}, \mathrm{H}+\mathrm{Al}, \mathrm{Al}, \mathrm{SB}$ e CTC de um Latossolo Vermelho distroférrico, com as concentrações foliares de $\mathrm{Ca}$, $\mathrm{Mg}, \mathrm{S}, \mathrm{Cu}, \mathrm{Fe}, \mathrm{Mn}$ e Zn da soja (Glycine max L.) cultivada em plantio direto. Foi instalada uma rede amostral, para a coleta de dados do solo e das plantas, com 100 pontos amostrais distribuídos, aletoriamente, em uma área de $7.980 \mathrm{~m}^{2}$. Os resultados da análise geoestatística mostraram que para os atributos avaliados houve dependência espacial. Os menores alcances observados foram os das constantes variações da área pelo preparo de um sistema de produção. Tanto linear quanto espacialmente, a CTC do solo foi bom indicador das concentrações foliares de Ca e de S da soja sob plantio direto.

Palavras-chave: cerrado; Glycine max (L.) Merrill; manejo do solo; nutrição de plantas.

\section{ABSTRACT}

\section{Spatial variability of soybean leaf nutrient concentrations related to chemical attributes of an Oxisol}

The study of soil and plant properties using geostatistics and the consequent generation of management maps result in the rational use of fertilizers and thus in the reduction of costs for producers. We aimed to evaluate the linear and spatial dependence of the attributes $\mathrm{Ca}, \mathrm{Mg}, \mathrm{H}+\mathrm{Al}, \mathrm{Al}, \mathrm{SB}$, and CTC of an Oxisol, with foliar concentrations of $\mathrm{Ca}$, $\mathrm{Mg}, \mathrm{Cu}, \mathrm{Fe}, \mathrm{Mn}$, and $\mathrm{Zn}$ of soybean (Glycine max L.) grown in no-tillage in the agricultural year 2013/2014, in Selvíria, MS, Brazil. A sampling network was installed to collect soil and plant data, with 100 sampling points randomly distributed in an area of $7,980 \mathrm{~m}^{2}$. The results of geostatistical analysis showed that, for the evaluated attributes, there was spatial dependence. The lower reaches observed were the constant variations of the area by the preparation of a production system. Both linearly and spatially, soil CTC was a good indicator of foliar concentrations of Ca and S of soybean under no-tillage.

Key words: cerrado; Glycine max (L.) Merrill; soil management; plant nutrition.

\footnotetext{
Submetido em 10/11/2015 e aprovado em 11/07/2017

${ }^{1}$ Trabalho elaborado na disciplina de Geoestatística ministrada na pós-graduação do curso de Agronomia da UNESP/Ilha Solteira.

${ }^{2}$ Universidade Estadual Paulista "Júlio de Mesquita Filho", Faculdade de Engenharia, Departamento de Fitossanidade, Engenharia Rural e Solos, Ilha Solteira, São Paulo, Brasil. rngazola@gmail.com; lenon_lovera@hotmail.com, thiagocelestrino@yahoo.com.br; raissa_dinalli@terra.com.br; montanari@agr.feis.unesp.br; langoisa@gmail.com

*Autor para correspondência: langoisa@ gmail.com
} 


\section{INTRODUÇÃO}

O Brasil é o maior exportador mundial de soja, tendo exportado, até o final de junho de 2017, mais de 61,9 milhões de toneladas, o que evidencia a importância socioeconômica desse grão para o País (Conab, 2017). Segundo a Companhia Nacional de Abastecimento (Conab, 2017), a safra brasileira de soja (Glycine max (L.) Merrill) de 2016/2017 apresentou produção de 113.923,1 mil toneladas, com incremento de $19,4 \%$ em relação à safra 2015/16. A maior área plantada com a oleaginosa, de 15193,6 mil hectares, ocorreu na Região Centro-Oeste.

Machado et al. (2004) concluíram que, em relação à soja, cultura que exporta grandes quantidades de nutrientes das áreas de plantio, a recomendação georreferenciada de adubos e corretivos assume importância, pelo fato de as produtividades dos talhões serem niveladas pelo potencial produtivo de cada parte do terreno e por esse tipo de manejo representar redução do custo da adubação, que atinge valores na faixa de 25 a $35 \%$ do custo total de produção da cultura. Neste contexto, a tendência é de integração de diversas fontes de dados, a fim de melhor gerenciar a produção agrícola, de maneira a reconhecer que as culturas e os solos não devem ser manejados individualmente e de forma homogênea (Dalchiavon et al., 2011).

Os atributos do solo desempenham importante papel no desenvolvimento das plantas; portanto, a variabilidade espacial dos seus valores deve ser bem conhecida, visando a minimizar os erros de amostragem e de manejo, pois o solo apresenta heterogeneidade, tanto vertical quanto horizontalmente (Montanari et al., 2010). Práticas conservacionistas, como o plantio direto, criam ambiente distinto daquele encontrado com o preparo convencional (resultante dos efeitos dos resíduos vegetais superficiais e da reduzida movimentação do solo). Portanto, os mapeamentos destes atributos em áreas agrícolas são fundamentais para as recomendações de práticas de manejo (Basso et al., 2011).

A estatística clássica assume que a variação das características do solo dentro das unidades amostrais não é correlacionada e que a média das amostras é o melhor estimador das características de solo, em qualquer local, na unidade amostral. Avanços tecnológicos na agropecuária têm mostrado a importância de se medir as variações, espacial e temporal, de propriedades que influem no rendimento das culturas, com o objetivo de otimizar o aproveitamento de recursos e diminuir custos, criando, assim, as zonas específicas de manejo. Com esse fim, temse o uso da geoestatística (Carvalho et al., 2002).

De acordo com Rodrigues Junior et al. (2011), zona de manejo ou zona específica de manejo é a subregião do campo que apresenta combinação de fatores limitantes de produtividade e de qualidade, para a qual se pode aplicar dose uniforme de insumos, o que facilita a aplicação das técnicas de agricultura de precisão (AP), posto que se podem empregar, no manejo das culturas, os mesmos sistemas utilizados na agricultura convencional.

Bernardi et al. (2004) constataram que o uso da geoestatística permitiu identificar a variabilidade espacial dos teores de nutrientes das folhas de soja cultivada em sistema plantio direto, sendo que, com base nos alcances dos semivariogramas, a grade de amostragem mais adequada para representar a variabilidade espacial dos teores de nutrientes das folhas de soja foi de $20 \times 20 \mathrm{~m}$.

Diante do exposto, o objetivo deste trabalho foi avaliar as dependências linear e espacial dos atributos químicos do solo com as concentrações dos macronutrientes secundários e dos micronutrientes catiônicos da soja, com o intuito de gerar zonas específicas de manejo por meio do uso da geoestatística.

\section{MATERIAL E MÉTODOS}

O experimento foi realizado na área experimental da Fazenda de Ensino, Pesquisa e Extensão da Faculdade de Engenharia de Ilha Solteira (FEIS/UNESP), localizada no município de Selvíria, MS, entre as latitudes de $20^{\circ} 18^{\prime} 05^{\prime \prime} \mathrm{S}$ e $20^{\circ} 18^{\prime} 28^{\prime \prime} \mathrm{S}$ e as longitudes de 52³9’02"O e 5240’28"O, no ano agrícola 2013/14. A precipitação pluvial média anual da área é de $1.300 \mathrm{~mm}$, enquanto a temperatura média é de $23,7^{\circ} \mathrm{C}$. O tipo climático é Aw, segundo Köppen, caracterizado como tropical úmido com estação chuvosa no verão e seca no inverno.

A malha experimental foi instalada em um Latossolo Vermelho distroférrico típico argiloso, com declive homogêneo de 0,025 $\mathrm{m} \mathrm{m}^{-1}$ (Embrapa, 2006). O solo vinha sendo utilizado com a sucessão das culturas do milho ou soja (verão) e feijão ou milho (inverno), há, pelo menos, 28 anos. Nos primeiros 15 anos, foi realizado o preparo convencional para condução das culturas mencionadas e, nos últimos 13 anos, adotou-se o plantio direto.

O espaçamento entre linhas da cultura da soja foi de $0,45 \mathrm{~m}$, com a densidade final de 16 plantas por metro na linha de semeadura. Na adubação de semeadura, foram aplicados $400 \mathrm{~kg} \mathrm{ha}^{-1}$ da formulação 08-28-16. Para o tratamento de sementes, foram utilizados o fungicida carboxina (200 g i.a. $100 \mathrm{~kg}^{-1}$ de sementes) e o inoculante líquido (Rhizobium sp.), com 3109 células por g de semente.

A determinação dos pontos de coleta de amostras de solo e de planta foi feita com malha estatística. Nas plantas foram determinadas as concentrações foliares dos macronutrientes secundários $(\mathrm{Ca}, \mathrm{Mg}$ e $\mathrm{S})$ e dos micronutrientes catiônicos ( $\mathrm{Cu}, \mathrm{Fe}, \mathrm{Zn}$ e $\mathrm{Mn}$ ); os do solo foram o Ca, Mg, H+Al, Al, SB, CTC e m. As concentrações foliares de $\mathrm{Ca}, \mathrm{Mg}$ e $\mathrm{S}$ foram determinadas, coletando-se o terceiro 
trifólio, e as determinações dos nutrientes foi realizada segundo a metodologia de Malavolta et al. (1997).

A grade georreferenciada foi confeccionada com 100 pontos, distribuídos aleatoriamente, de forma a cobrir a área escolhida para amostragem $\left(7.980 \mathrm{~m}^{2}\right)$. Suas coordenadas originaram planilha de dados de latitude e longitude de cada ponto no espaço. Com o receptor GPS (Garmin Etrex ${ }^{\circledR)}$ alimentado por essa planilha de coordenadas, os pontos foram materializados na área do experimento.

As amostras para determinação dos atributos do solo foram coletadas individualmente em cada ponto amostral, que foi constituído da coleta dos dados das plantas posicionadas no centro e de suas circunvizinhas. A época de coleta de amostras de solo foi a última semana de janeiro de 2013, durante a fase de florescimento da soja. As amostras de solo para essas determinações foram coletadas com trado de caneca, com diâmetro interno de $0,08 \mathrm{~m} \mathrm{e}$ altura de 0,20 m, nas profundidades de 0,00-0,10 e 0,10-0,20 $\mathrm{m}$. A determinação da análise química do solo foi realizada segundo a metodologia descrita por Raij et al. (2001).

Para cada atributo estudado, foi efetuada a análise descritiva, auxiliada pela estatística clássica. Utilizando-se o SAS (Schlotzhaver \& Littel, 1997), calcularam-se média, mediana, moda, valores mínimo e de máximo, desvio padrão, coeficiente de variação, curtose e assimetria. Em seguida, foram identificados os pontos anômalos (outliers), conforme identificação no gráfico de ramos e folhas, efetuando-se a substituição deles pelo valor médio dos circunvizinhos presentes na malha geoestatística. Também foi efetuada a análise da distribuição de frequência dos dados. Desta forma, para testar a hipótese de normalidade, ou de lognormalidade dos atributos (x), foi utilizado o teste de Shapiro e Wilk, a 1\%, em que a estatística W testa a hipótese nula, a qual julga ser a amostra proveniente de população com distribuição normal. Assim, no caso de dados transformados na forma logarítmica $(\mathrm{y}=\ln \mathrm{x}), \mathrm{W}$ testa a hipótese nula de que os valores yi provêm de uma distribuição normal, isto é, os dados não transformados (xi) ajustam-se a distribuição lognormal.

Foi montada a matriz de correlação de Pearson, entre todos os atributos estudados (solo e planta) para o conjunto de amostras, por intermédio do SAS e EXCEL.

Para cada atributo, foi analisada a dependência espacial pelo cálculo do semivariograma, com bases nos pressupostos de estacionariedade da hipótese intrínseca, usando-se o software geoestatístico $\mathrm{GS}^{+}$, versão 7.0 (Gamma Design Software, 2004). Foram ajustados semivariogramas simples a todos os atributos estudados da planta e do solo.

Os ajustes dos semivariogramas simples em função de seus modelos foram efetuados prioritariamente pela seleção inicial de: a) o maior coeficiente de determinação $\left(\mathrm{r}^{2}\right)$; b) a menor soma dos quadrados dos desvios (SQD), e c) o maior avaliador do grau da dependência espacial
(ADE). A decisão final do modelo que representou o ajuste foi realizada pela validação cruzada, assim como para a definição do tamanho da vizinhança que proporcionou a melhor malha de krigagem, realizadas por meio da krigagem em blocos. Para cada atributo, foram estimados o efeito pepita $\left(\mathrm{C}_{\mathrm{o}}\right)$, o alcance $\left(\mathrm{A}_{\mathrm{o}}\right)$ e o patamar $\left(\mathrm{C}_{\mathrm{o}}+\mathrm{C}\right)$.

\section{RESULTADOS E DISCUSSÃO}

Dos atributos pesquisados (Tabela 1), somente as concentrações foliares (Ca, Mg e S) e a CTC do solo apresentaram baixa variabilidade. A acidez potencial do solo e as concentrações foliares de $\mathrm{Mn}$ e de $\mathrm{Zn}$ apresentaram variabilidade média; soma de bases e teores de $\mathrm{Ca}$ e $\mathrm{Mg}$ do solo mostraram variabilidade alta e Al, saturação por alumínio e concentrações foliares de $\mathrm{Cu}$ e Fe, muito alta. Segundo Pimentel-Gomes \& Garcia (2002), a variabilidade de um atributo pode ser classificada segundo a magnitude de seu coeficiente de variação (CV), quando o CV for $\leq 10 \%$, baixa; $10 \%<\mathrm{CV} \leq 20 \%$, média, $20 \%<\mathrm{CV} \leq 30 \%$, alta e CV > $30 \%$, muito alta.

Avaliando-se o coeficiente de variação da produtividade do feijoeiro, correlacionada com atributos químicos de um Latossolo Vermelho distroférrico sob Plantio direto, Dalchiavon et al. (2011) observaram alta variabilidade para os teores de $\mathrm{Ca}, \mathrm{Mg}$ e SB, semelhantemente ao observado neste estudo.

Dentre os atributos analisados, constatou-se distribuição de frequência do tipo normal para saturação por alumínio, na camada de 0,00-0,10 m (m1), capacidade de troca catiônica, na camada de 0,10-0,20 m (CTC2) e para as concentrações foliares de $\mathrm{Ca}, \mathrm{Mg}, \mathrm{Mn}$ e Zn. Segundo Sfredo et al., 1986, as concentrações foliares de $\mathrm{Ca}, \mathrm{Mg}$ e $\mathrm{Zn}$ (Tabela 1) são tidas como suficientes para a cultura. Já, para o Mn (Tabela 1), a concentração é considerada muito alta. Segundo o mesmo autor, a elevada concentração de $\mathrm{Mn}$ da folha da soja é devida ao baixo $\mathrm{pH}$ que deve predominar na área de estudo, em cujo solo a realização da calagem diminui a disponibilidade desse nutriente.

As correlações entre os pares dos atributos químicos do solo e as concentrações de nutrientes da folha da soja foram baixas, porém, com valores significativos, a 5\%, entre a CTC do solo e as concentrações foliares de Ca e S (Tabela 2).

As cargas negativas da CTC efetiva do solo são neutralizadas, principalmente, pelas bases $\left(\mathrm{Ca}^{2+}, \mathrm{Mg}^{2+}, \mathrm{K}^{+}, \mathrm{Na}^{+} \mathrm{e}\right.$ $\mathrm{NH}_{4}^{+}$, pelo alumínio $\left(\mathrm{Al}^{3+}\right)$ e, também, pelos cátions de hidrogênio $\left(\mathrm{H}^{+}\right)$(Novais \& Mello, 2007). Segundo Malavolta (2006), o íon cálcio, nas condições de solos não muito ácidos, é o cátion predominante no complexo de troca.

Nesse sentido, solos com elevada CTC apresentam maior capacidade de retenção dos cátions (K, Ca e Mg) e, consequentemente, maior fornecimento desses cátions 


Medidas estatísticas descritivas

\begin{tabular}{|c|c|c|c|c|c|c|c|c|c|c|}
\hline \multirow[t]{2}{*}{ Atributos $^{(a)}$} & \multirow{2}{*}{ Média } & \multirow{2}{*}{ Mediana } & \multicolumn{2}{|c|}{ Valor } & \multirow{2}{*}{$\begin{array}{l}\text { Desvio } \\
\text { Padrão }\end{array}$} & \multicolumn{3}{|c|}{ Coeficiente } & \multicolumn{2}{|c|}{ Probabilidade do teste (b) $^{(b)}$} \\
\hline & & & Mínimo & Máximo & & Variação (\%) & Curtose & Assimetria & $\operatorname{Pr}<w$ & DF \\
\hline \multicolumn{11}{|c|}{ Concentrações foliares } \\
\hline \multicolumn{11}{|l|}{$\left(\mathrm{g} \mathrm{kg}^{-1}\right)$} \\
\hline $\mathrm{Ca}$ & 10,64 & 10,60 & 8,00 & 13,70 & 1,07 & 10,00 & 0,398 & 0,404 & 0,223 & NO \\
\hline $\mathrm{Mg}$ & 3,79 & 3,80 & 3,10 & 4,60 & 0,31 & 8,17 & $-0,311$ & 0,200 & 0,256 & NO \\
\hline$S$ & 1,94 & 1,90 & 1,70 & 2,20 & 0,12 & 6,34 & $-0,185$ & 0,533 & 0,0001 & IN \\
\hline \multicolumn{11}{|l|}{$\left(\mathrm{mg} \mathrm{kg}^{-1}\right)$} \\
\hline $\mathrm{Cu}$ & 7,3 & 6,0 & 3,0 & 16,0 & 3,11 & 42,68 & 0,754 & 1,229 & 0,0001 & IN \\
\hline $\mathrm{Fe}$ & 171,32 & 159,00 & 100,00 & 325,00 & 5,19 & 30,29 & 0,789 & 1,127 & 0,0001 & IN \\
\hline $\mathrm{Mn}$ & 255,63 & 254,50 & 156,00 & 360,00 & 40,39 & 15,80 & $-0,140$ & 0,296 & 0,426 & $\mathrm{NO}$ \\
\hline $\mathrm{Zn}$ & 29,7 & 29,0 & 18,0 & 43,0 & 5,12 & 17,24 & $-0,218$ & 0,311 & 0,362 & NO \\
\hline
\end{tabular}

Atributos químicos do solo

\begin{tabular}{|c|c|c|c|c|c|c|c|c|c|c|}
\hline \multicolumn{11}{|c|}{$\left(\mathrm{mmol}_{\mathrm{c}} \mathrm{dm}^{-3}\right)$} \\
\hline $\mathrm{Ca}_{1}$ & 9,97 & 10 & 4 & 18 & 2,96 & 29,8 & $-0,099$ & 0,466 & 0,023 & IN \\
\hline $\mathrm{Ca}_{2}$ & 10,08 & 10 & 5 & 18 & 2,95 & 29,3 & 0,168 & 0,667 & 0,001 & IN \\
\hline $\mathrm{Mg}_{1}^{2}$ & 8,81 & 8,5 & 5 & 18 & 2,35 & 26,7 & 1,630 & 0,842 & 0,003 & IN \\
\hline $\mathrm{Mg}_{2}$ & 8,08 & 8 & 4 & 13 & 1,98 & 24,48 & $-0,255$ & 0,438 & 0,002 & IN \\
\hline $\mathrm{H}+\mathrm{Al}_{1}$ & 38,39 & 38 & 25 & 50 & 4,24 & 11,1 & 0,582 & $-0,083$ & 0,034 & IN \\
\hline $\mathrm{H}+\mathrm{Al}_{2}$ & 36,61 & 38 & 28 & 47 & 4,64 & 12,69 & $-0,460$ & 0,092 & 0,005 & IN \\
\hline $\mathrm{Al}_{1}$ & 5,71 & 6 & 0 & 13 & 2,45 & 42,9 & 0,127 & 0,390 & 0,022 & IN \\
\hline $\mathrm{Al}_{2}$ & 2,67 & 3 & 0 & 6 & 1,65 & 61,86 & $-0,716$ & $-0,046$ & 0,001 & IN \\
\hline $\mathrm{SB}_{1}$ & 20,57 & 20,30 & 10,8 & 38,8 & 5,67 & 27,59 & 0,597 & 0,557 & 0,019 & IN \\
\hline $\mathrm{SB}_{2}$ & 19,43 & 18,20 & 9,5 & 34,3 & 5,38 & 27,68 & 0,152 & 0,759 & 0,001 & IN \\
\hline $\mathrm{CTC}_{1}^{2}$ & 58,98 & 58,9 & 41,8 & 74,7 & 5,40 & 9,15 & 1,613 & $-0,098$ & 0,021 & $\mathrm{TN}$ \\
\hline $\mathrm{CTC}_{2}$ & 55,95 & 55,5 & 45,9 & 71,8 & 4,85 & 8,68 & 0,271 & 0,492 & 0,243 & NO \\
\hline \multicolumn{11}{|l|}{$(\%)$} \\
\hline $\mathrm{m}_{1}$ & 22,48 & 21 & 0 & 52 & 10,70 & 47,61 & $-0,262$ & 0,432 & 0,101 & $\mathrm{NO}$ \\
\hline $\mathrm{m}_{2}$ & 13,03 & 12 & 0 & 32 & 8,56 & 65,70 & $-0,895$ & 0,073 & 0,004 & IN \\
\hline
\end{tabular}

(a) Concentrações foliares: $\mathrm{Ca}, \mathrm{Mg}, \mathrm{S}, \mathrm{Cu}, \mathrm{Fe}, \mathrm{Mn}$ e $\mathrm{Zn}$, respectivamente, cálcio, magnésio, enxofre, cobre, ferro, manganês e zinco; Atributos químicos do solo: Ca1, Ca2, Mg1, Mg2, H+Al1, H+Al2, Al1, A12, SB1, SB2, CTC1, CTC2, m1 e m2, respectivamente, cálcio, magnésio, acidez potencial, alumínio, soma de bases, capacidade de troca catiônica e saturação por alumínio, nas profundidades de 0-0,10 m (1) e 0,10-0,20 m (2); (b) DF = distribuição de frequência, sendo NO, LN, TN, TL e IN, respectivamente, do tipo normal, log-normal, tendendo a normal, tendendo a lognormal e indeterminada. 
Tabela 2: Matriz de correlação linear simples entre alguns atributos químicos das camadas de 0,00-0,10 e 0,10-0,20 m, de um Latossolo Vermelho distroférrico, e de concentrações foliares de nutrientes da soja

Coeficiente de correlação ${ }^{(\mathrm{b})}$

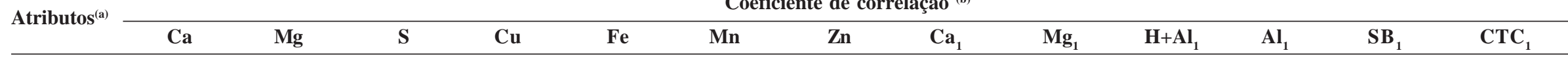

\begin{tabular}{|c|c|c|c|c|c|c|c|c|c|c|c|c|c|}
\hline$\overline{\mathrm{Mg}}$ & $0,59^{* *}$ & - & - & - & - & - & - & - & - & - & - & - & - \\
\hline $\mathrm{S}$ & $0,39^{* *}$ & $0,06^{\mathrm{ns}}$ & - & - & - & - & - & - & - & - & - & - & - \\
\hline $\mathrm{Cu}$ & $-0,11^{\mathrm{ns}}$ & $-0,11^{\mathrm{ns}}$ & $0,08^{\text {ns }}$ & - & - & - & - & - & - & - & - & - & - \\
\hline $\mathrm{Fe}$ & $-0,14^{\mathrm{ns}}$ & $-0,02^{\mathrm{ns}}$ & $0,01^{\mathrm{ns}}$ & $0,01^{\mathrm{ns}}$ & - & - & - & - & - & - & - & - & - \\
\hline $\mathrm{Mn}$ & $0,19^{*}$ & $0,10^{\mathrm{ns}}$ & $0,23^{*}$ & $0,12^{\mathrm{ns}}$ & $-0,27^{* * *}$ & - & - & - & - & - & - & - & - \\
\hline $\mathrm{Zn}$ & $0,16^{\mathrm{ns}}$ & $0,04^{\mathrm{ns}}$ & $0,36^{* *}$ & $0,41^{* *}$ & $0,12^{\mathrm{ns}}$ & $0,14^{\mathrm{ns}}$ & - & - & - & - & - & - & - \\
\hline $\mathrm{Ca}_{1}$ & $0,19^{*}$ & $-0,03^{\mathrm{ns}}$ & $0,24^{*}$ & $0,06^{\mathrm{ns}}$ & $-0,13^{\text {ns }}$ & $-0,11^{\mathrm{ns}}$ & $0,06^{\mathrm{ns}}$ & - & - & - & - & - & - \\
\hline $\mathrm{Mg}_{1}$ & $0,06^{\mathrm{ns}}$ & $-0,07^{\mathrm{ns}}$ & $0,14^{\mathrm{ns}}$ & $0,09^{\mathrm{ns}}$ & $-0,03^{\text {ns }}$ & $-0,23^{*}$ & $-0,06^{\mathrm{ns}}$ & $0,83^{* *}$ & - & - & - & - & - \\
\hline $\mathrm{H}+\mathrm{Al}_{1}$ & $0,17^{\mathrm{ns}}$ & $0,27^{* *}$ & $0,16^{\mathrm{ns}}$ & $-0,08^{\mathrm{ns}}$ & $-0,02^{\mathrm{ns}}$ & $0,06^{\mathrm{ns}}$ & $0,17^{\mathrm{ns}}$ & $-0,24^{*}$ & $-0,25^{*}$ & - & - & - & - \\
\hline $\mathrm{Al}_{1}$ & $-0,01^{\mathrm{ns}}$ & $0,08^{\mathrm{ns}}$ & $-0,01^{\mathrm{ns}}$ & $-0,21^{*}$ & $-0,07^{\mathrm{ns}}$ & $0,12^{\mathrm{ns}}$ & $0,03^{\mathrm{ns}}$ & $-0,60^{* *}$ & $-0,58^{* *}$ & $0,67^{* *}$ & - & - & - \\
\hline $\mathrm{SB}_{1}$ & $0,13^{\mathrm{ns}}$ & $-0,06^{\mathrm{ns}}$ & $0,16^{\mathrm{ns}}$ & $0,08^{\mathrm{ns}}$ & $-0,08^{\mathrm{ns}}$ & $-0,15^{\mathrm{ns}}$ & $0,01^{\mathrm{ns}}$ & $0,94^{* *}$ & $0,92^{* * *}$ & $-0,29^{* * *}$ & $-0,63^{* *}$ & - & - \\
\hline $\mathrm{CTC}_{1}$ & $0,24^{*}$ & $0,14^{\mathrm{ns}}$ & $0,24^{*}$ & $0,02^{\mathrm{ns}}$ & $-0,07^{\mathrm{ns}}$ & $-0,08^{\mathrm{ns}}$ & $0,10^{\mathrm{ns}}$ & $0,68^{* *}$ & $0,60^{* *}$ & $0,38^{* *}$ & $-0,15^{\mathrm{ns}}$ & $0,68^{* *}$ & - \\
\hline \multirow[t]{2}{*}{$\mathrm{m}_{1}$} & $-0,03^{\mathrm{ns}}$ & $0,06^{\mathrm{ns}}$ & $-0,06^{\mathrm{ns}}$ & $-0,22^{*}$ & $-0,03^{\mathrm{ns}}$ & $0,12^{\mathrm{ns}}$ & $-0,01^{\mathrm{ns}}$ & $-0,76^{* *}$ & $-0,74^{* *}$ & $0,55^{* *}$ & $0,95^{* *}$ & $-0,80^{* * *}$ & $-0,39^{* *}$ \\
\hline & $\mathbf{C a}$ & Mg & $\mathbf{S}$ & $\mathbf{C u}$ & $\mathrm{Fe}$ & Mn & $\mathbf{Z n}$ & $\mathrm{Ca}_{2}$ & $\mathbf{M g}_{2}$ & $\mathrm{H}+\mathrm{Al}_{2}$ & $\mathbf{A l}_{2}$ & $\mathrm{SB}_{2}$ & $\mathrm{CTC}_{2}$ \\
\hline $\mathrm{Ca}_{2}$ & $0,06^{\mathrm{ns}}$ & $-0,12^{\mathrm{ns}}$ & $0,19^{\mathrm{ns}}$ & $0,08^{\mathrm{ns}}$ & $-0,09^{\mathrm{ns}}$ & $-0,27^{* *}$ & $0,04^{\mathrm{ns}}$ & - & - & - & - & - & - \\
\hline $\mathrm{Mg}_{2}$ & $0,13^{\mathrm{ns}}$ & $-0,04^{\mathrm{ns}}$ & $0,19^{\mathrm{ns}}$ & $0,08^{\mathrm{ns}}$ & $-0,16^{\mathrm{ns}}$ & $-0,17^{\mathrm{ns}}$ & $0,06^{\mathrm{ns}}$ & $0,80^{* *}$ & - & - & - & - & - \\
\hline $\mathrm{H}+\mathrm{Al}_{2}$ & $0,31^{* *}$ & $0,18^{\mathrm{ns}}$ & $0,08^{\mathrm{ns}}$ & $-0,20^{*}$ & $0,04^{\mathrm{ns}}$ & $0,10^{\mathrm{ns}}$ & $0,04^{\mathrm{ns}}$ & $-0,53^{* *}$ & $-0,51^{* *}$ & - & - & - & - \\
\hline $\mathrm{Al}_{2}$ & $-0,02^{\mathrm{ns}}$ & $-0,01^{\mathrm{ns}}$ & $-0,09^{\mathrm{ns}}$ & $-0,10^{\mathrm{ns}}$ & $-0,09^{\mathrm{ns}}$ & $0,29^{* *}$ & $0,01^{\mathrm{ns}}$ & $-0,60^{* *}$ & $-0,60^{* *}$ & $0,61^{* *}$ & - & - & - \\
\hline $\mathrm{SB}_{2}$ & $0,19^{\mathrm{ns}}$ & $-0,03^{\mathrm{ns}}$ & $0,25^{* *}$ & $0,07^{\mathrm{ns}}$ & $-0,09^{\mathrm{ns}}$ & $-0,25^{* *}$ & $0,10^{\mathrm{ns}}$ & $0,85^{* *}$ & $0,92^{* *}$ & $-0,45^{* *}$ & $-0,67^{* *}$ & - & - \\
\hline $\mathrm{CTC}_{2}$ & $0,45^{* *}$ & $0,12^{\mathrm{ns}}$ & $0,32^{* *}$ & $-0,10^{\mathrm{ns}}$ & $-0,07^{\mathrm{ns}}$ & $-0,18^{\mathrm{ns}}$ & $0,14^{\mathrm{ns}}$ & $0,43^{* * *}$ & $0,49^{* *}$ & $0,40^{* *}$ & $-0,13^{\mathrm{ns}}$ & $0,61^{* * *}$ & - \\
\hline $\mathrm{m}_{2}$ & $-0,03^{\mathrm{ns}}$ & $0,03^{\text {ns }}$ & $-0,10^{\mathrm{ns}}$ & $-0,12^{\mathrm{ns}}$ & $-0,02^{\mathrm{ns}}$ & $0,31^{* *}$ & $-0,02^{\mathrm{ns}}$ & $-0,74^{* *}$ & $-0,73^{* *}$ & $0,61^{* *}$ & $0,96^{* *}$ & $-0,79^{* *}$ & $-0,25^{*}$ \\
\hline
\end{tabular}

(a) Concentrações foliares: $\mathrm{Ca}, \mathrm{Mg}, \mathrm{S}, \mathrm{Cu}, \mathrm{Fe}, \mathrm{Mn}$ e $\mathrm{Zn}$, respectivamente, cálcio, magnésio, enxofre, cobre, ferro, manganês e zinco; $\mathrm{Atributos} \mathrm{químicos} \mathrm{do} \mathrm{solo:} \mathrm{Ca}_{1}, \mathrm{Mg}_{1}, \mathrm{H} \mathrm{Al}_{1}, \mathrm{Al}_{1}, \mathrm{SB}_{1}, \mathrm{CTC}_{1}$ e $\mathrm{m}_{1}$, respectivamente, cálcio, magnésio, acidez potencial, alumínio, soma de bases, capacidade de troca catiônica e saturação por alumínio, nas profundidades de $0,00-0,10 \mathrm{~m}(1) ; \mathrm{Ca}_{2}, \mathrm{Mg}_{2}, \mathrm{H} \mathrm{Al}_{2}, \mathrm{Al}_{2}, \mathrm{SB}_{2}, \mathrm{CTC}_{2}$ e $\mathrm{m}_{2}$, respectivamente, cálcio, magnésio, acidez potencial, alumínio, soma de bases, capacidade de troca catiônica e saturação por alumínio, nas profundidades de $0,10-0,20 \mathrm{~m}$ (2); (b) * Significativo a 5\%, ** Significativo a $1 \%$ 
para a planta, tendo em vista menores perdas por lixiviação. Em contrapartida, menor capacidade de troca aniônica (CTA), ou seja, menor retenção de ânions, dentre eles o $\mathrm{SO}^{2-}$, resulta em maior mobilidade do $\mathrm{SO}_{4}^{2-}$, que descerá em profundidade acompanhado de cátions ( $\mathrm{K}, \mathrm{Ca}$ e $\mathrm{Mg}$ ), estimulando o maior crescimento radicular, o que faz que a raiz explore maior volume de solo, absorvendo maior quantidade do elemento.

$\mathrm{O}$ ânion $\mathrm{SO}_{4}^{2-}$ é menos retido na camada arável do solo que os cátions $\mathrm{Ca}^{2+} \mathrm{e} \mathrm{Mg}^{2+}$. Segundo Raij (2011), três fatores dificultam a permanência desse ânion nessa camada: a presença de maiores teores de matéria orgânica, que aumentam as cargas negativas do solo, portanto, repelindo os sulfatos; a frequente prática da calagem, que promove a liberação dos sulfatos adsorvidos, e a prática da adubação fosfatada, com o que os íons fosfatos têm preferência pelas posições de troca que seriam ocupadas por sulfatos.

Com os valores mínimo e máximo da CTC1, de 41,8 e $74,7 \mathrm{mmol}_{\mathrm{c}} \mathrm{dm}^{-3}$, da Tabela 1 , foram estimadas as concentrações foliares de $\mathrm{Ca}(9,8-11,4)$ e de $\mathrm{S}(1,8-2,0)$. Para o Ca, essa faixa é tida como suficiente (média) e, para o $\mathrm{S}$, de média a baixa, segundo a interpretação de análises de tecido de folhas de soja proposta por Sfredo et al., 1986. Em função da CTC2, a faixa concentrações de $\mathrm{Ca}(9,6-12,2)$ foi tida como suficiente (média) e a de $\mathrm{S}(1,8-2,1)$, como de média a baixa (Figura 1).
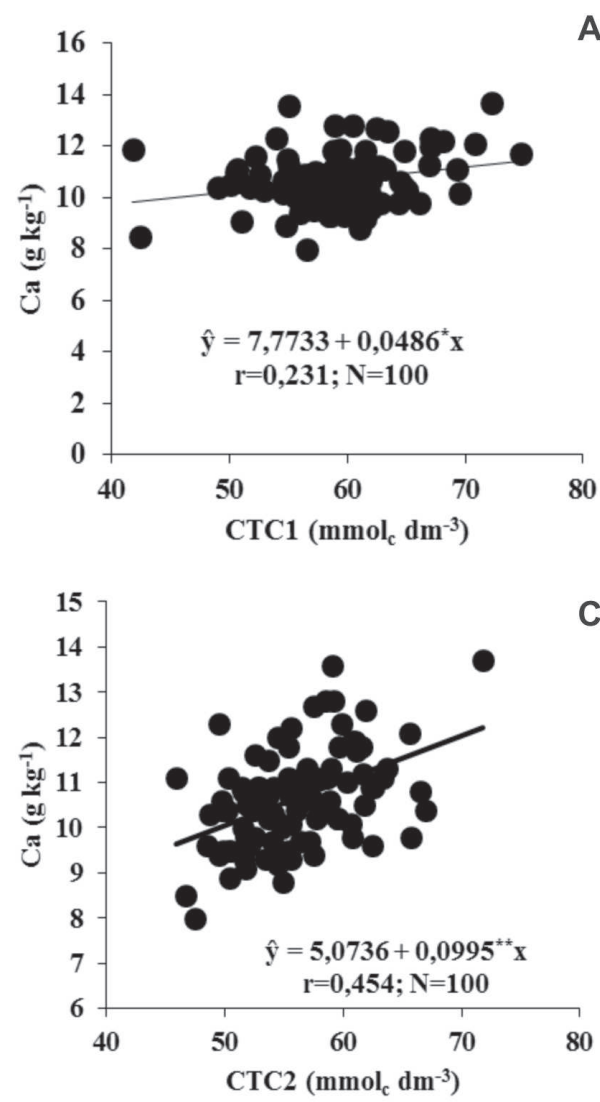

Somente para $\mathrm{Cu}, \mathrm{Zn}$ e soma de bases da camada de 0,10-0,20 m não se observou dependência espacial (Tabela 3). Os coeficientes de determinação espacial $\left(\mathrm{r}^{2}\right)$ para os atributos que apresentaram dependência, observados nos semivariogramas, decresceram na seguinte ordem: Mn (0,990), Fe (0,964), CTC2 (0,962), H+Al (0,942), S (0,882), Mg2 (0,873), H+Al2 (0,815), m1 (0,788), CTC1 (0,787), Ca1 (0,781), SB1 (0,773), $\operatorname{Mg}(0,736), \mathrm{m} 2(0,698), \mathrm{Al1}(0,694)$, $\mathrm{Ca}(0,667), \mathrm{Mg} 1(0,638), \mathrm{Al} 2(0,628)$ e Ca2 $(0,303)$. A concentração foliar de $\mathrm{Mn}$ apresentou aleatoriamente a menor dispersão dos lags em torno do modelo exponencial ajustado, o melhor semivariograma, com ADE médio de $82,0 \%$ e alcance $\left(\mathrm{A}_{\mathrm{o}}\right)$ de $21,0 \mathrm{~m}$.

Reichert et al. (2008) estudaram a variabilidade espacial de alguns atributos químicos de um Planossolo e sua relação com a produtividade de soja e separaram o alcance em dois grupos distintos, o primeiro sendo representado pelos atributos com semivariograma gaussiano e alcance menor que $67 \mathrm{~m}$, dentre eles o Ca, $\mathrm{Mg}$ e CTC (49,8; 66,5 e 46,2), amostrados na camada de 0,00-0,15 do solo. Neste estudo, o modelo dos semivariogramas desses atributos foi exponencial, com alcances de 24,3; 17,4 e 19,5 m, (Ca1, Mg1 e CTC1, respectivamente). O maior alcance verificado por esses autores deve-se provavelmente ao processo de sistematização das áreas de plantio

A

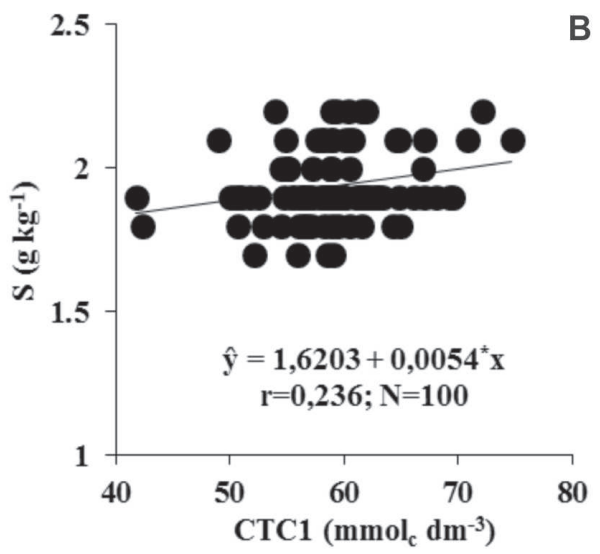

B

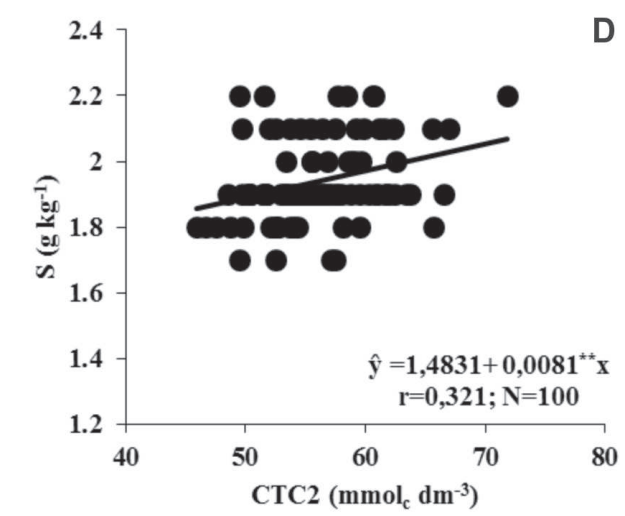

Figura 1: Concentrações de Ca e S foliar da soja, em função da CTC, de 0,00-0,10 m (a e b, respectivamente) e em função da CTC, de 0,10-0,20 m (c e d, respectivamente), de um Latossolo Vermelho distroférrico. 
(nivelamento), o que resulta em maior homogeneização dos atributos químicos do solo da área.

Dalchiavon et al. (2011) avaliaram, também, em um Latossolo Vermelho distroférrico, a variabilidade espacial da produtividade do feijoeiro, correlacionada com atributos químicos do solo, sendo constatados alcances de 13,7; 16,0 e 38,8 m (Ca, Mg e CTC, respectivamente), semelhantes ao constatado neste estudo.

O segundo grupo proposto (Reichert et al., 2008) foi composto pelos atributos com semivariograma exponencial com alcance menor que $40 \mathrm{~m}$, sendo eles $\mathrm{Al}$ e m (16,0 e 15,4 m, respectivamente). Neste estudo, verificaram-se alcances de 24,0 e 34,5 m (Al1 e m1, respectivamente), com modelo de semivariograma também exponencial.

Segundo a interpretação proposta, para o ADE, por (Dalchiavon et al., 2012): a) ADE < 20\% = variável espacial de muito baixa dependência; b) $20 \% \leq \mathrm{ADE}<40 \%=$ baixa dependência; c) $40 \% \leq \mathrm{ADE}<60 \%=$ média dependência; d) $60 \% \leq \mathrm{ADE}<80 \%=$ alta dependência) e $80 \% \leq$ $\mathrm{ADE}<100 \%=$ muito alta dependência, todos os atribu- tos, com exceção do Fe, Mg2, H+Al1 e H+Al2 (alta, média, média e média, respectivamente) apresentaram dependência espacial classificada como muito alta, com predomínio do modelo do semivoriograma do tipo exponencial, exceto para concentração foliar de S, Fe, Mn e H+Al1 do solo (esféricos). Nesse sentido, as distâncias entre pontos utilizadas na malha geoestatística deste estudo foram suficientes para avaliar a variabilidade espacial dos atributos químicos do solo, nas profundidades de 0,00-0,10 e 0,100,20 m, das concentrações dos macronutrientes secundários e dos micronutrientes (Fe e $\mathrm{Mn}$ ).

Os valores para alcances das concentrações foliares dos macronutrientes secundários estão compreendidos entre 13,8 e 18,9 m; os do Fe e Mn, entre 21,0 e 55,7 m e, para os atributos químicos do solo ( $\mathrm{Ca}, \mathrm{Mg}, \mathrm{H}+\mathrm{Al}, \mathrm{Al}, \mathrm{SB}, \mathrm{CTC}$ e m), entre 17,4 e 45,8 m (camada de 0,00-0,10 m) e entre 17,1 e 77,4 m (camada de 0,10-0,20 m). Esse parâmetro fornecido pela análise geoestatística é de fundamental importância, pois representa a distância em que uma variável regionalizada apresenta continuidade espacial, sendo que a acima desta

Tabela 3: Parâmetros dos semivariogramas ajustados para alguns atributos químicos de um Latossolo Vermelho distroférrico e de concentrações foliares de nutrientes da soja

\begin{tabular}{|c|c|c|c|c|c|c|c|c|}
\hline \multirow{3}{*}{ Atributo } & \multirow{3}{*}{ Modelo } & \multicolumn{5}{|c|}{ Parâmetros do ajuste } & \multirow{2}{*}{\multicolumn{2}{|c|}{ ADE }} \\
\hline & & \multirow{2}{*}{$\mathrm{C}_{\mathrm{o}}$} & \multirow{2}{*}{$\mathrm{C}_{\mathrm{o}}+\mathrm{C}$} & \multirow{2}{*}{$\mathbf{A}_{\mathrm{o}}(\mathbf{m})$} & \multirow{2}{*}{$\mathbf{r}^{2}$} & \multirow{2}{*}{ SQR } & & \\
\hline & & & & & & & $\%$ & Classe \\
\hline \multicolumn{9}{|c|}{ Gama (h) simples - planta } \\
\hline $\mathrm{Ca}$ & $\exp (201)$ & $8,1.10^{-2}$ & $8,79.10^{-1}$ & 18,6 & 0,667 & $2,0.10^{-2}$ & 90,8 & muito alta \\
\hline $\mathrm{Mg}$ & $\exp (186)$ & $8,7.10^{-3}$ & $7,6.10^{-2}$ & 18,9 & 0,736 & $1,29.10^{-4}$ & 88,5 & muito alta \\
\hline S & esf (181) & $1,1.10^{-3}$ & $1,1.10^{-2}$ & 13,8 & 0,882 & $6,7 \cdot 10^{-7}$ & 90,2 & muito alta \\
\hline $\mathrm{Cu}$ & epp & 20,38 & - & - & - & - & - & - \\
\hline $\mathrm{Fe}$ & esf (209) & 1097 & 3228 & 55,7 & 0,964 & $9,98.10^{4}$ & 66,1 & alta \\
\hline $\mathrm{Mn}$ & esf (202) & 232,0 & 1288,0 & 21,0 & 0,990 & $2,21.10^{3}$ & 82,0 & muito alta \\
\hline $\mathrm{Zn}$ & epp & 49,1 & - & - & - & - & - & - \\
\hline \multicolumn{9}{|c|}{ Gama (h) simples - solo } \\
\hline $\mathrm{Ca} 1$ & $\exp (205)$ & 1,26 & 8,24 & 24,3 & 0,781 & 1,72 & 84,7 & muito alta \\
\hline $\mathrm{Ca} 2$ & $\exp (216)$ & 0,88 & 7,63 & 20,7 & 0,303 & 10,30 & 88,5 & muito alta \\
\hline $\operatorname{Mg} 1$ & $\exp (206)$ & 0,61 & 4,70 & 17,4 & 0,638 & 0,52 & 87,0 & muito alta \\
\hline $\mathrm{Mg} 2$ & $\exp (189)$ & 1,74 & 3,48 & 77,4 & 0,873 & 0,18 & 50,0 & média \\
\hline $\mathrm{H}+\mathrm{A} 11$ & esf (199) & 7,48 & 16,20 & 45,8 & 0,942 & 2,53 & 53,8 & média \\
\hline $\mathrm{H}+\mathrm{Al} 2$ & $\exp (195)$ & 10,39 & 22,85 & 51,3 & 0,815 & 12,4 & 54,5 & média \\
\hline Al1 & $\exp (217)$ & 0,84 & 5,80 & 24,0 & 0,694 & 1,32 & 85,5 & muito alta \\
\hline $\mathrm{A} 12$ & $\exp (199)$ & 0,31 & 2,39 & 19,5 & 0,628 & 17,9 & 86,3 & muito alta \\
\hline SB1 & $\exp (188)$ & 4,32 & 29,28 & 20,1 & 0,773 & 16,3 & 85,2 & muito alta \\
\hline SB2 & epp & 49,70 & - & - & - & - & - & - \\
\hline CTC1 & $\exp (181)$ & 0,65 & 22,69 & 19,5 & 0,787 & 12,1 & 97,1 & muito alta \\
\hline CTC2 & $\exp (209)$ & 1,20 & 24,23 & 38,7 & 0,962 & 5,39 & 95,0 & muito alta \\
\hline $\mathrm{m} 1$ & $\exp (192)$ & 17,80 & 109,80 & 34,5 & 0,788 & 527 & 83,8 & muito alta \\
\hline $\mathrm{m} 2$ & $\exp (182)$ & 7,70 & 57,97 & 17,1 & 0,698 & 64,2 & 86,7 & muito alta \\
\hline
\end{tabular}

Concentrações foliares: $\mathrm{Ca}, \mathrm{Mg}, \mathrm{S}, \mathrm{Cu}, \mathrm{Fe}, \mathrm{Mn}$ e $\mathrm{Zn}$, respectivamente, cálcio, magnésio, enxofre, cobre, ferro, manganês e zinco; Atributos químicos do solo: Ca1, Ca2, Mg1, Mg2, H+Al1, H+Al2, Al1, Al2, SB1, SB2, CTC1, CTC2, m1 e m2, respectivamente, cálcio, magnésio, acidez potencial, alumínio, soma de bases, capacidade de troca catiônica e saturação por alumínio, nas profundidades de 0-0,10 m (1) e 0,10$0,20 \mathrm{~m}$ (2). Parênteses sucedendo o modelo $=$ número de pares no primeiro lag. exp = exponencial; epp = efeito pepita puro e esf $=$ esférico. $\mathrm{SQR}=$ soma dos quadrados dos resíduos. $\mathrm{ADE}=$ avaliador da dependência espacial. 
distância, o comportamento espacial da variável passa a ser totalmente aleatório (Lemos Filho et al., 2008).

Esse resultado leva à sugestão de que, em trabalhos futuros que visem a avaliar a concentração foliar de Ca, $\mathrm{Mg}, \mathrm{S}, \mathrm{Fe}$ e Mn na cultura da soja, utilizem-se malhas geoestatísticas e distâncias entre pontos iguais aos deste estudo, pois os valores dos alcances a serem utilizados nos pacotes geoestatísticos, que alimentarão os pacotes computacionais empregados na agricultura de precisão, em geral, deverão estar compreendidos entre esses valores, por representarem a distância dentro da qual os valores de um determinado atributo são próximos entre si.

Após os ajustes dos semivariogramas dos atributos químicos do solo, das concentrações foliares dos nutrientes da soja, foram construídos mapas de krigagem, que são de grande importância para a agricultura de precisão, pois são definidas zonas específicas de manejo da fertilidade do solo, o que leva à otimização do uso dos insumos, pois sua aplicação a taxas variadas permite a redução de seus custos, além de homogeneizar a fertilidade do solo.

Visualiza-se, no mapa de krigagem, maiores valores de CTC1 (> 62,9 $\mathrm{mmol}_{\mathrm{c}} \mathrm{dm}^{-3}$ ) no sentido Oeste-Leste (Figura $2 \mathrm{a}$ ) e predomínio de áreas com teores maiores que $56,6 \mathrm{mmol}_{\mathrm{c}} \mathrm{dm}^{-3}$. Para camada de 0,10-0,20 m, os maiores valores da CTC2 (> 64,2 $\left.\mathrm{mmol}_{\mathrm{c}} \mathrm{dm}^{-3}\right)$ ocorreram na região leste, os menores $\left(47,6 \mathrm{mmol}_{\mathrm{c}} \mathrm{dm}^{-3}\right)$ predominando na região norte do mapa (Figura 2b). Valores maiores de $\mathrm{Ca}$ $\left(11,2\right.$ e $\left.12,2 \mathrm{~g} \mathrm{~kg}^{-1}\right)$ e $\mathrm{S}\left(1,95\right.$ e 2,06 $\left.\mathrm{g} \mathrm{kg}^{-1}\right)$ ocorreram nas regiões central e leste dos mapas (Figuras $2 \mathrm{c}$ e d, respectivamente), como verificado para a CTC1 (sentido OesteLeste) e CTC2 (região Leste).
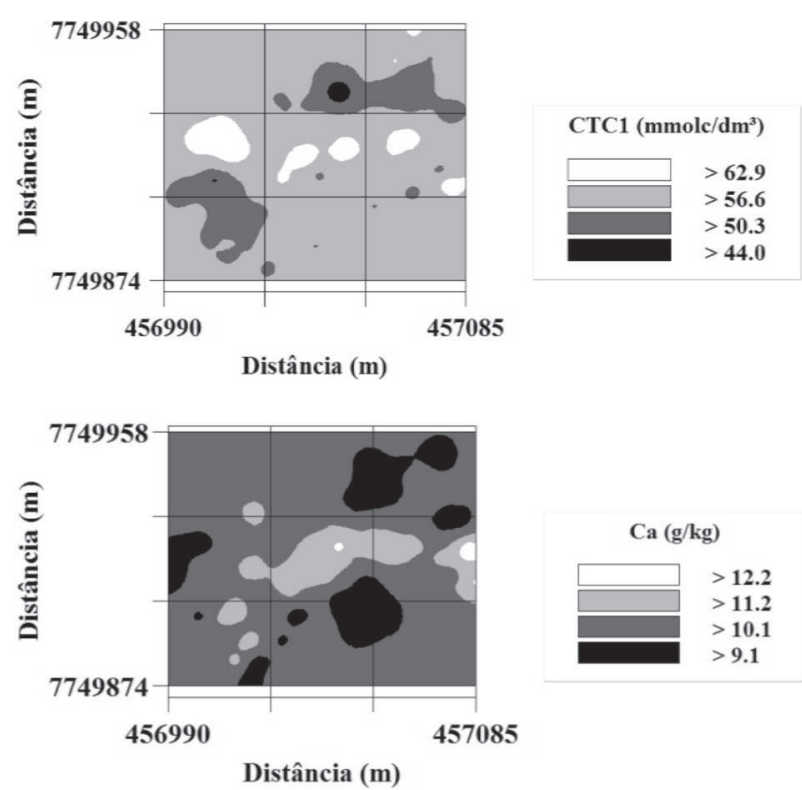

Conforme apresentado na Figura 2, a região central dos mapas de krigagem apresentou maiores valores de CTC, em profundidade, e concentração foliar de Ca e S, não apresentando distribuição espacial homogênea; porém, a recomendação ao produtor é realizar o manejo da adubação nas extremidades do mapa de krigagem, pois apresenta valores menores que os da região central do mapa. Soares (2013), analisando a viabilidade financeira do plantio de soja, com a agricultura de precisão, encontrou maior produção $(17,65 \%)$ e economia $(5,56 \%)$ do que com a agricultura tradicional.

\section{CONCLUSÕES}

Espacialmente, duas zonas específicas de manejo foram discriminadas, a primeira com elevada capacidade de troca catiônica (CTC) e a segunda com os baixos valores; assim, o tipo de manejo deve ser realizado conforme as diferentes zonas indicadas no mapa de krigagem.

Os atributos químicos do solo e as concentrações foliares de $\mathrm{Ca}, \mathrm{Mg}$, S e Mn apresentam dependência espacial classificada como muito alta, com modelo dos semivariogramas ajustados predominantemente do tipo exponencial.

Tanto linear quanto espacialmente, a CTC do solo, nas profundidades de 0,00-0,10 e 0,10-0,20 m, destaca-se como um potencial indicador das concentrações foliares de Ca e $\mathrm{S}$ da soja cultivado sob plantio direto.

Para este trabalho, o teor de magnésio da camada de 0,01-0,20 m foi o atributo dependente que apresentou o maior alcance $(77,4 \mathrm{~m})$ e, assim, recomenda-se que em fu-
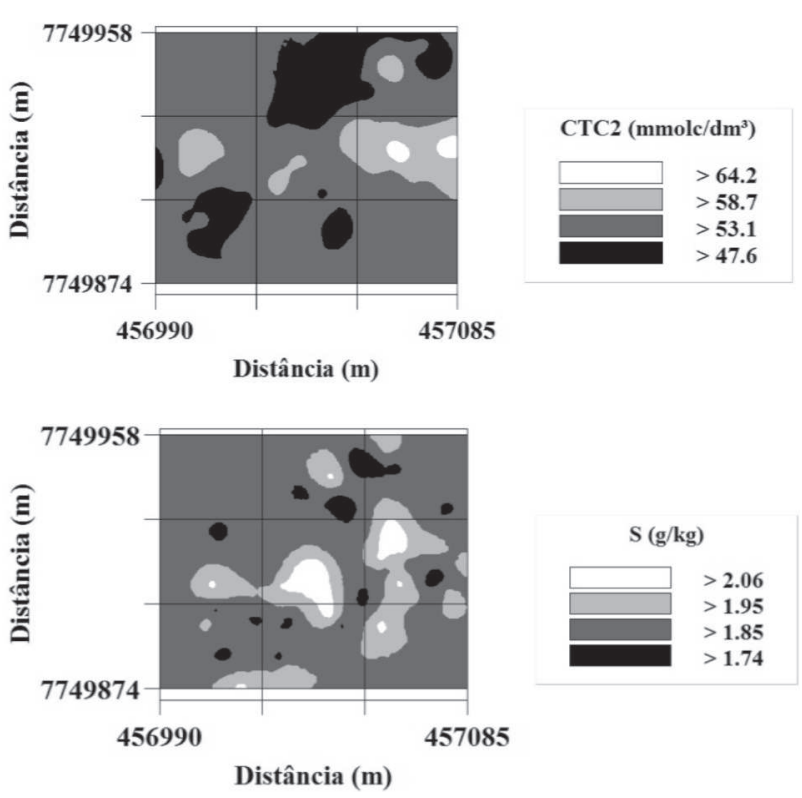

Figura 2: Mapas de krigagem da capacidade de troca catiônica em ambas as profundidades e das concentrações foliares de Ca (cálcio) e S (enxofre) da soja (a, b, c e d, respectivamente, em um Latossolo Vermelho distroférrico sob plantio direto. 
turas pesquisas as distâncias entre as avaliações não sejam inferiores a esse valor.

\section{AGRADECIMENTOS}

Os autores agradecem aos Primeiros ProjetosFUNDUNESP, processo número: 0235/001/14-PROPe/CDC, pelo apoio financeiro.

\section{REFERÊNCIAS}

Basso FC, Andreotti M, Carvalho MPE, Lodo BN \& Montanari R (2011) Correlação linear e espacial entre a produtividade e o teor de proteína bruta do guandu anão e os atributos de um Latossolo. Revista Brasileira de Ciências Agrárias, 6:521530 .

Bernardi ACC, Carmo CAFS, Machado PLOA, Silva CA, Valencia LIO \& Meirelles MSP (2004) Avaliação Especializada do Estado Nutricional da Soja. In: Machado PLOA, Bernardi ACC \& Silva CA (Eds.) Agricultura de precisão para o manejo da fertilidade do solo em sistema plantio direto. Rio de Janeiro, Embrapa Solos. p.131-152.

Carvalho JRP, Silveira PM \& Vieira SR (2002) Geoestatística na determinação da variabilidade espacial de características químicas do solo sob diferentes preparos. Pesquisa Agropecuária Brasileira, 37:1151-1159.

Conab - Companhia Nacional de Abastecimento (2017) Acompanhamento da safra brasileira - Grãos. Safra 2016/17. Disponível em: <http://www.conab.gov.br/OlalaCMS/uploads/arquivos/ 17_06_08_09_02_48_boletim_graos_junho_2017.pdf > Acessado em: 12 de junho de 2017.

Dalchiavon FC, Carvalho MP, Andreotti M \& Montanari R (2012) Variabilidade espacial de atributos da fertilidade de um Latossolo Vermelho Distroférrico sob Sistema Plantio Direto. Revista Ciência Agronômica, 43:453-461.

Dalchiavon FC, Carvalho MP, Freddi OS, Andreotti M \& Montanari R (2011) Variabilidade espacial da produtividade do feijoeiro correlacionada com atributos químicos de um Latossolo Vermelho Distroférrico sob sistema de semeadura direta. Bragantia, 70:908-16.

Embrapa - Empresa Brasileira de Pesquisa Agropecuária (2006) Sistema brasileiro de classificação de solos. $2^{\mathrm{a}}$ ed. Rio de Janeiro, Embrapa SPI. 306p.

Pimentel-Gomes F \& Garcia CH (2002) Estatística aplicada a experimentos agronômicos e florestais. Piracicaba, Fealq. 309p.

Gamma Design Software (2004) GS ${ }^{+}$: Geostatistics for environmental sciences. $7^{\text {th }}$ ed. Plainwell, Gamma Desing Software. 159 p.

Lemos Filho LCA, Oliveira EL, Faria MA \& Andrade LAB (2008) Variação espacial da densidade do solo e matéria orgânica em área cultivada com cana-de-açúcar (Saccharum officinarum L.). Revista Ciência Agronômica, 39:193-202.

Machado PLOA, Bernardi ACC, Silva CA, Carmo CAFS, Meirelles MSP \& Manzatto CV (2004) Estudo de caso em agricultura de precisão: manejo de lavoura de soja na região de campos gerais, PR. In: Machado PLOA, Bernardi ACC \& Silva CA (Eds.) Agricultura de precisão para o manejo da fertilidade do solo em sistema plantio direto. Rio de Janeiro, Embrapa Solos. p.93113.

Malavolta E, Vitti GC \& Oliveira AS (1997) Avaliação do estado nutricional das plantas: princípios e aplicações. $2^{\mathrm{a}}$ ed. Piracicaba, Potafós. 319p.
Malavolta E (2006) Manual de nutrição mineral de plantas. São Paulo, Agronômica Ceres. 631p.

Montanari R, Carvalho MP, Andreotti M, Dalchiavon FC, Lovera LH \& Honorato MAO (2010) Aspectos da produtividade do feijão correlacionados com atributos físicos do solo sob elevado nível tecnológico de manejo. Revista Brasileira de Ciência de Solo, 34:1811-1822.

Novais RF \& Mello JWV (2007) Relação solo-planta. In: Novais RF, Alvarez V VH, Barros NF, Fontes RLF, Cantarutti RB \& Neves JCL (Eds.) Fertilidade do Solo. Viçosa, SBCS. p.133-204.

Raij BV, Andrade JC, Cantarella H \& Quaggio JA (2001) Análise química para avaliação da fertilidade de solos tropicais. Campinas: IAC. 285 p.

Raij VB (2011) Fertilidade do solo e manejo de nutrientes. Piracicaba, IPNI. 420p.

Reichert JM, Dariva TA, Reinert DJ \& Silva VR (2008) Variabilidade espacial de Planossolo e produtividade de soja em várzea sistematizada: análise geoestatística e análise de regressão. Ciência Rural, 38:981-988.

Rodrigues Junior FA, Vieira LB, Queiroz DM \& Santos NT (2011) Geração de zonas de manejo para cafeicultura empregando-se sensor SPAD e análise foliar. Revista Brasileira de Engenharia Agrícola e Ambiental, 15:778-787.

Schlotzhaver SD \& Littell RC (1997) SAS System for elementary statistical analysis. $2^{\text {nd }}$ ed. Cary, SAS Institute. 905p.

Sfredo GJ, Lantmann AF, Campo RJ \& Borkert CM (1986) Soja: nutrição mineral, adubação e calagem. Londrina, EmbrapaCNPSo. $51 \mathrm{p}$.

Soares IPM (2013) Análise de viabilidade financeira do plantio de soja utilizando a agricultura de precisão. Trabalho de Conclusão de Curso. Centro Universitário Municipal de Franca, São Paulo. 59 p. 